\title{
Adverse Events Following Administration of DTwP Containing Vaccines in Albanian Children from 2003 to 2015
}

\author{
Irsida Mehmeti, (MS) \\ Catholic University “Our Lady of Good Counsel”, Albania \\ Silva Bino, (PhD) \\ Erida Nelaj, (PhD) \\ Eugena Tomini \\ Institute of Public Health, Tirana, Albania
}

doi: 10.19044/esj.2016.v12n33p40 URL:http://dx.doi.org/10.19044/esj.2016.v12n33p40

\begin{abstract}
The Albanian vaccination program started in 1960. DTwP vaccine was introduced to the immunization schedule since the beginning and was produced in the country until the establishment of EPI (Expanded Program on Immunization - EPI) in 1993, at the Department of Epidemiology of the Institute of Public Health (IPH). From that time the vaccines were procured by UNICEF. Until 2003, the reporting of adverse events after vaccination was not made through a standard reporting form. The adverse events reporting system was implemented in 2003 through the introduction of individual reporting forms to the primary health care centers. Although the surveillance system was a passive one, the collection of data by IPH was made on monthly base and when a signal was detected further investigations were undertaken. The pertussis component of Diphtheria, Tetanus, Pertussis (DTwP) vaccine is the whole inactivated cell of Bordetella Pertussis. The aim of this study is to analyze the rate of adverse events following DTwP administration in Albanian children from 2003 to 2015.
\end{abstract}

Keywords: Adverse events, vaccination, DTwP, rates

\section{Introduction}

Whole cell pertussis vaccines (wP) were firstly produced and worldwide adopted during the 1940s and were widely accepted despite the local and systemic adverse reactions following its administration (Mortimer, 1994). Since that year diphtheria and tetanus toxoids have been combined with pertussis antigens and used as a combination DTwP. In Albania, this 
vaccine was introduced to the immunization schedule since the beginning of vaccination program all over the country in 1960, but untill 2009 it was administered as a single inoculation to the Albanian children in a three primary dose and one booster dose. In 2009, the Pentavalent vaccine was introduced in the immunization schedule and DTwP was co-administered with HepB and Hib vaccines in a single formulation (DTP-HepB-Hib). This Pentavalent vaccine replaced the traditional DTwP in all the doses. The primary doses are administered at 2, 4 and 6 months of age while the booster dose is administered at 24 months of age. At the same day, the child receives also the OPV(Oral Polio Vaccine). Studies and clinical trials have shown that the co-administration of more than one antigen does not increase the rate of adverse events of the single one (Bar-On, Goldberg, Hellmann, \& Leibovici, 2012; Decker, Edwards, \& Bogaerts, 2008). Administering multiple antigens into a single injection reduces pain, local reactions, distress of the child and also limits the costs of maintaining the cold chain.

There have been a lot of controversies regarding adverse events after whole cell pertussis vaccines since it is known as one of the most reactogenic vaccines. These vaccines which are suspected to kill the B. pertussis organisms might cause frequently minor reactions, infrequently transient with moderate severity reactions and rarely serious but self-limited reactions. The concerns about safety issues of wP led to the development of acellular pertussis (aP) vaccine in the late 1980s which nowadays is used in almost all industrialized countries (Institute of Medicine [IOM], 1991). In Albania, wP vaccine it is still used, but aP can be obtained through private vaccine suppliers. This study was undertaken to compare rates and frequency of local and systemic reactions after administration of DTwP-containing vaccines to children of 2, 4, 6 and 24 months of age. Most of the events were common and there were minor reactions like local reactions but only rare serious events. Local reactions included erythema, swelling, pain and tenderness at the injection site. Systemic events included generalized urticaria, rash, drowsiness, persistent crying, encephalopathy, anaphylaxis and convulsions. No deaths were reported. The most used Pentavalent vaccine was Quinvaxem (Korea) but also other brands like Bio-Farma (Indonesia), GlaxoSmithKline (Belgium) and Indian brands were reported. Taking in consideration the WHO information sheets on rates of adverse events of each vaccine, it is possible that most of the adverse events reported after Pentavalent vaccine administration were related to DTP component (World Health Organization [WHO], 2014). The limitations of this study include the nature of the AEFI system. Passive surveillance system leads to underreporting, over-reporting of minor events in some occasions, errors in classification and diagnosis of the events and missing details about some of 
the events which could make possible a more accurate conclusion about the relationship between the vaccine and the adverse event.

The aim of this study is: i) to determine whether the adverse events after DTwP administration are higher than the rates reported for the DTP vaccine in the information sheets published by World Health Organization and, ii) to compare the pattern and frequency of these events with those reported in other studies of this type.

\section{Material and Methods}

A retrospective analysis of the adverse events database was undertaken using IBM-SPSS ver.21 statistics program. The AEFI database was analyzed for adverse events reported following DTwP containing vaccines. A total of 275 adverse events were reported after immunization with DTP-containing vaccines during a period of 13 years (2003-2015) and for a total of 1,825,528 administered doses. Various statistics test were used to calculate the difference in group categories, ratios, and frequencies. A pvalue $<0.05$ was considered statistically significant.

\section{Results}

Several studies and clinical trials comparing adverse events after DTwP or DT vaccine administration have demonstrated that most of the side effects associated with DTP administration are related to the whole cell pertussis component of the vaccine, although it is largely, but not solely, responsible for reactions occurring after administration of the combined DTwP vaccine (Gold, Kempe, \& Osbourn, 1999 ; Greco et. al., 1996 ; Braun, Salive, Chen, \& Ellenberg, 2000). The differences are more evident when they regard the benign adverse effects such as fever, reactions at the injection site and fussiness (Gold et al., 1999 ; Greco et al., 1996). Almost $45 \%$ $(124 / 275)$ of the events were reported in females, $35 \%(97 / 275)$ in males and in $20 \%(54 / 275)$ of the cases data about gender was missing. The overall mean age of vaccinee manifesting an AEFI after DTP administration was 6 months (CI 95\%: 5,4-7,1).

\section{Reporting Rates after DTwP-containing vaccines throughout years}

The mean annual reporting rate after DTwP-containing vaccines is 14.4 cases $/ 100.000$ administered doses $(p=0.023$, CI 95\%: 2,25-25,63). The distribution of the AEFI rates is not the same throughout years $(p=0.045)$ but shows two peak levels. In the Figure1 the first peak level is observed in 2004 which corresponds to the second year after implementation of AEFI reporting system. This is due to increased awareness and trainings of health care workers by campaigns undertaken by the Institute of Public Health. The second peak is observed during 2010, the second year after the introduction 
of the Pentavalent vaccine to the national immunization schedule. This is known as the Webber effect; a tendency to report any adverse event after the introduction of a new vaccine into the immunization schedule (Australian Government, 2008). This period is followed by a constant level of reporting rates of adverse events after DTwP administration.

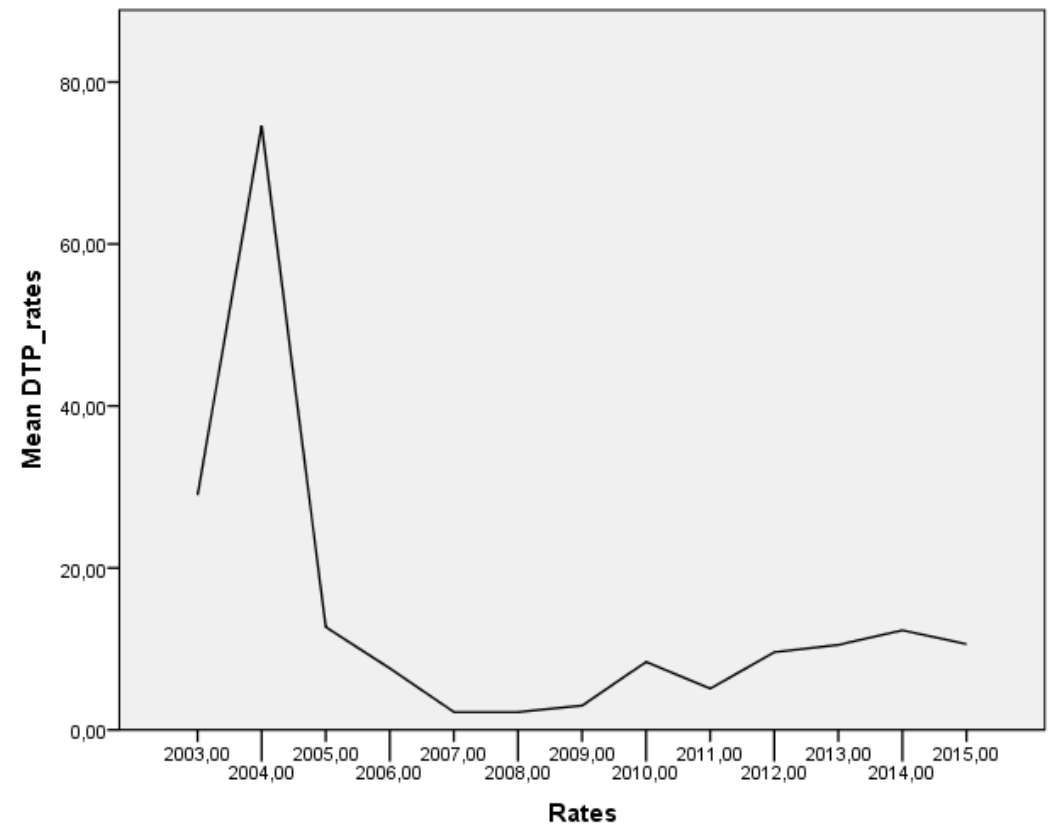

Figure 1. Reporting rates of adverse events after administration of DTwP containing vaccines from 2003 to 2015.

\section{Dose number}

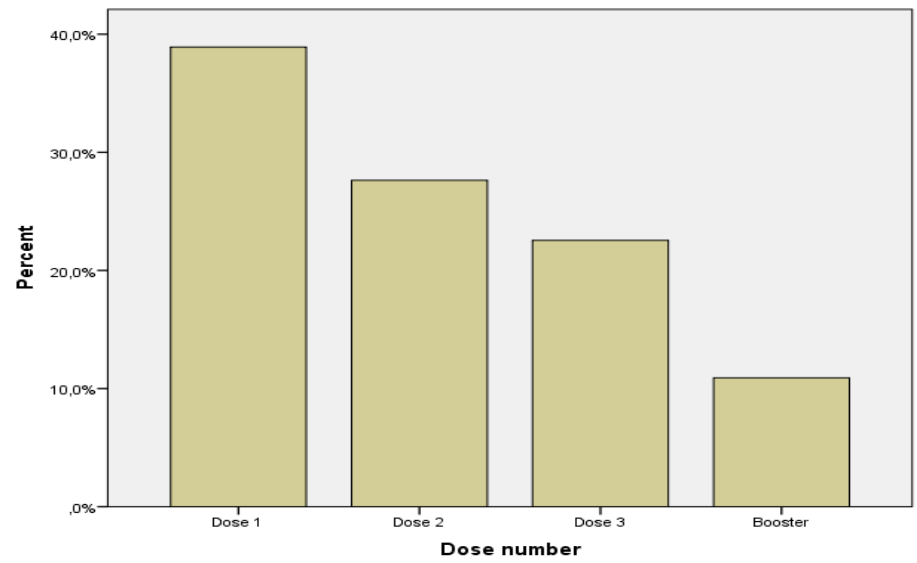

Figure 2. Frequency of adverse events reported after administration of DTwP-containing vaccines versus dose number 
About 38,8\%(107/275) of the reactions occurred after the first administered dose of DTP vaccine. However, $80.3 \%$ (86/107) of these reported adverse events after the first dose administration are just events of fever over $38{ }^{0} \mathrm{C}$. 43,8\% (28/64) of the local reactions also occurred after the first dose. Persistent crying occurred most frequently with the initial dose and less often thereafter (7/11). More severe reactions like convulsions occurred at the same frequency after the first and second dose (6/17 and 5/17 cases respectively). Anaphylaxis occurred more frequently after the first dose administration (5/8). In 4 out of 6 cases the convulsions occurred after the first dose. According to WHO, children who experienced local reactions after pertussis vaccine have an enhanced probability of experiencing the same reaction with a following dose (Deloria et al., 1995). Two studies conclude that the frequency of local reactions tends to increase with the number of doses while systemic reactions, with the exception of fever, may diminish with subsequent doses (Cody, Baraff, Cherry, Marcy \& Manclark, 1981 ; Pollock, Miller, Mortimer, \& Smith, 1984). This is not observed in our analysis.

\section{Uncommon serious reactions}

Cody et al., reported that DTwP vaccine is associated with febrile seizures (0.06 \% of the vaccinees) and the risk for seizures after DTwP administration is higher in children with personal or family histories of convulsions (Cody et al., 1981). However simple febrile convulsions in children are considered to be benign with no evidence for risk of epilepsy. There were no cases of Hypotonic-Hyporesponsive-Episode (HHE) reported after DTwP immunization in Albanian children. The Cody and colleagues study detected an incidence of $\mathrm{HHE}$ of $0.06 \%$ after immunization of children with DTwP-vaccine (Cody et al.,1981). Serious reactions constitute $30 \%(84 / 275)$ of all cases reported after administration of DTwP-containing vaccines in Albania. The frequency of non-serious reaction is significantly higher than that of serious reactions $\left(p=0.000, \chi^{2}=41,6\right)$. The definition of serious reaction is based on the definition made by WHO: Serious adverse events following immunization are considered those that result in death; are life-threatening; require in-patient hospitalization or prolongation of existing hospitalization; result in persistent or significant disability/incapacity, or; are a congenital anomaly/birth defect (WHO, 2000). 96,8 \% of the non-serious cases healed after treatment compared with $89,4 \%$ of the serious group. $48,8 \%$ of the serious cases occurred during 2004, which corresponds to the second year of implementation of AEFI reporting system. After this year the frequency of reporting of serious AEFI has been constant throughout the years. 


\begin{tabular}{|c|c|c|c|}
\hline \multirow{2}{*}{ AEFI severity } & \multicolumn{3}{|c|}{ Health condition after treatment } \\
\cline { 2 - 4 } & Not healed & Healed & Total \\
\hline Non Serious & 5 & 149 & 154 \\
\hline & $3,20 \%$ & $96,80 \%$ & $100,00 \%$ \\
\hline Serious & 7 & 59 & 66 \\
\hline & $10,60 \%$ & $89,40 \%$ & $100,00 \%$ \\
\hline Total & 12 & 208 & 220 \\
\hline & $5,50 \%$ & $94,50 \%$ & $100,00 \%$ \\
\hline
\end{tabular}

Table 1. Outcome of treatment of serious and non-serious cases

\section{Anaphylaxis}

Because of the controversy in defining the anaphylaxis and lacking of a widely accepted definition of this term, there is variability in the data reporting this type of adverse effect (Ruggeberg et al., 2007). It is not known whether the reported event as anaphylaxis is referred to the immediate hypersensitivity reaction (type I anaphylaxis) or to a life-threatening clinical event. Taking in consideration the levels of certainty of Brighton Collaboration case definition, which is conform with the definition of European Academy of Allergy and Clinical Immunology (EAACI's) and the American Academy of Pediatrics', the definition of anaphylaxis in this study analyses the cardiovascular and/or respiratory involvement in presence of skin reactions and multisystem findings (Johansson et al., 2001). Anaphylaxis is a rare event in infancy and the background rates are not wellknown (Ruggeberg et al., 2007). The Committee to Review Adverse Effects of Vaccines in 2012 concluded that the evidence convincingly supports a causal relationship between tetanus toxoid vaccine and anaphylaxis (IOM, 1991). In this study the mean value of rate of anaphylaxis after DTwP administration to Albanian children is $0,45 / 100.000$ doses of DTwP administered ( $p=0,023$ CI: $0.06-0,73$ ) and a normal distribution of rates throughout years. In the WHO vaccine rates information's sheets of DTwP vaccine it is reported a rate of 0.13 (CI; 0.003 to 0.71 ) cases per 100,000 administered doses ( Bohlke et al., 2003). Most of the cases occurred after the first dose administration (5/8) and data suggest that this might be related to the presence of IgG antibodies transmitted from the mother. Generalized urticaria was reported in 5 cases, 1 of which reported as anaphylaxis, while rash was reported in 9 cases, angioedema in 4 cases, cyanosis in 6 cases and erythema in 6 cases. However it should be specified that the occurrence of these symptoms without a definite cause is not rare in infancy and their frequency after DTwP immunization has already been reported (WHO, 2014). 


\section{Encephalopathy, Encephalitis and Seizures}

There have been ongoing discussions about relationship between DTwP containing vaccines and encephalopathy (Fenichel, 1982). Although the terms encephalopathy and encephalitis are used interchangeably to indicate a set of symptoms like alterations in behavior or level of consciousness, convulsions, headache and focal neurological deficits, the term encephalitis implies an inflammatory reaction within the brain. Conversely, the term encephalopathy does not and it is frequently accompanied by febrile or non-febrile seizures although the latter alone is not a diagnosis for the former (Sejvar et al., 2007). Convulsions are motor manifestations of seizures (Ray et.al., 2006). Due to the variability of clinical symptoms and signs of the disease, the difficulty to distinguish between the two brain pathologies and the absence of a precise definition, it is difficult to give a definitive answer regarding the reporting rates of neurological adverse effects after DTwP vaccination in Albanian children. Seizures are much more common in children than is encephalopathy and it is probable that the majority of cases reported as encephalopathy might be acute symptomatic seizures. Although the report of Institute of Medicine in 1994 concluded that "the balance of evidence is consistent with a causal relationship between DTwP and chronic nervous system dysfunction in children whose serious acute neurological illness occurred within 7 days of a DTwP vaccination", recent studies do not confirm an association between DTwP and acute encephalopathy (Ray et.al., 2006). Moreover, increasing sophisticated diagnostic tools have revealed other causes, including Daveret syndrome, for almost all of the severe neurological syndromes that follow childhood vaccination and have been previously erroneously attributed to them (Ray et.al., 2006).

In our study, there has been in total 3 cases of encephalitis and the reporting rates has been 0,7 cases/100,000 of DTwP administered doses. In one case encephalitis was accompanied by convulsions and in another case with persistent crying and vomiting. Only one case was followed up at the moment of reporting but there is no follow up study. While the mean value of convulsions rate has been 0,95 cases/100,000 doses of DTwP vaccine administered ( $p=0.003$, CI: $0,31-1,42)$ there has been in total 17 cases of convulsions, 10 of which accompanied by febrile convulsions and in 4 cases by cyanosis . Only 3 cases with convulsions were being followed up at the moment of reporting. The other cases had no neurological impairment. It should be noted that all of these rates for encephalopathy after DTwP vaccination are significantly less than the risk for encephalopathy after B. pertussis infection itself ( Rantala \& Uhari, 1989). 


\title{
Persistent crying
}

Various types of studies like case reports, case series and controlled epidemiological studies have concluded that persistent crying after DTwP vaccination is probably caused by pain, local reaction, and fever (Bonhoeffer et al., 2013). The Committee to review adverse effects of vaccines in 1991 concluded that the evidence indicates a cause-effect relationship between the pertussis component of DTwP vaccine and protracted, inconsolable, or highpitched crying or screaming (IOM, 1991). The rate of persistent crying varies with type and dose of vaccine and immunization site. Since in this study the duration of persistent crying is not reported and neither the crying continuity, it is difficult to meet the Brighton Collaboration case definition for persistent crying (Bonhoeffer et al., 2013). However the rate of reporting is 0,6 cases per 100,000 administered doses. There have been 11 cases of persistent crying reported. In 9 cases fever was also reported and in 1 case convulsions and cyanosis and the children were all recovered after receiving treatment. $7 / 11$ of the cases occurred after the first dose administration .This is consistent with the DTwP rates information sheets of WHO and Multicenter Acellular Pertussis Trial (WHO, 2014; Decker \& Edwards , 1996).

\section{Common minor reactions}

Other researchers reported that minor local reactions, as redness, swelling, and pain at the site of injection, occur in about half of DTwP recipients (Cody et al., 1981). These reactions as well as minor systemic reactions such as fever, irritability, and drowsiness occur five times more frequently after DTwP than after DT vaccine administration (Cody et al., 1981). The frequency of common reactions after DTwP administration vary among the lots and manufacturers, while local reaction rates vary with the number of prior DTwP injections (Baraff, Cody \& Cherry, 1984).

\begin{abstract}
Abscess
There are in total 6 cases of abscess reported, 4 of which have occurred after administration of the first dose. Three of them were accompanied by fever while in the other two edema and erythema was reported. None of these cases meets the first level of diagnostic certainty of Brighton Collaboration because laboratory confirmation was not developed (Kohl et al., 2007). Local reactions like redness and tenderness have been associated with infectious abscesses which are also accompanied by fever. The sterile abscesses (cold abscess) are typically not accompanied by fever or lymphadenopathy (Kohl et al., 2007). All the cases, but one, were recovered without sequelae after receiving an adequate treatment.
\end{abstract}




\section{Local reactions}

This is the second most common group of adverse events reported after the fever. Local reactions constitute $11,3 \%$ of all adverse effects reported after DTwP vaccination. Limb pain was reported in 4 cases, local redness in 6 cases, local swelling in 9 cases and local pain in 1 case. Although there is no standardized definition, a "local reaction" is any description of morphological or physiological change at or near the injection site (Gidudu, 2008). The rate of local reactions depend on various factors like technique and methods of vaccine administration, manufacturing lot, storage conditions of the vaccine, genetic predisposition and route of administration. 24/31 of the cases resolved after ambulatory treatment while $7 / 31$ of the cases resolved after hospital treatment. 41,9\% of local reactions occurred after first dose and 22,6 \% after booster dose. This is inconsistent with the Cody and colleagues study which concludes that local reactions increased in frequency with successive doses. Local reactions are usually attributed to the B. pertussis-containing component (Cody et al., 1981).

\section{Rash}

There are many morphologies of rash which describe mucocutaneous changes after vaccination. It is important to report the color, number, size, morphology and anatomic distribution of the observed skin lesions (Beigel et.al., 2007). Rash was reported in 9 cases, generalized urticaria in 5 cases, local urticaria in 4 cases and cyanosis in 7 cases. 90,5\% of the cases recovered without any sequelae. Anyway the committee to review the adverse events of vaccines concluded that there is insufficient evidence to indicate a causal relation between DTwP vaccine or the $B$. pertussis component of DTwP vaccine and any kind of rash (IOM, 1991).

\section{Fever}

Fever is defined as the endogenous elevation of at least one measured body temperature of $\geq 38{ }^{\circ} \mathrm{C}$ and is distinguished from hyperthermia which is a body warming caused by external environmental factors (Bouchama \& Knochel, 2002). Fever was the most frequently reported adverse event after DTwP administration. Although the accuracy of measurement and errors in measurement method are not specified and this might influence the reporting rates. Mean fever rate is 12 cases/100.000 doses administered. The fever rates does not differ significantly throughout years but there is a significant increase in $2004(112 / 234$ of the cases, $p=0.046)$, the second year after implementation of AEFI reporting system. 80,3 \% of the cases with fever were classified as non-serious while 25,9 \% as serious reactions. Fever over $40{ }^{\circ} \mathrm{C}$ was reported in $3 \%$ of the cases $(7 / 234)$. The number of healed cases 
is higher than the number of non-healed cases $(\mathrm{p}=0.000$ ). The fever working group at Brighton Collaboration case definitions conclude that fever following administration of an inactivated component or live vaccine may be temporally associated with, but is not necessarily the result of the administration of a vaccine (Marcy et al., 2004). It should be recognized that background rates of fever are high. The value of $\geq 38{ }^{\circ} \mathrm{C}$ is accepted as reflecting an abnormal elevation of temperature, irrespective of device, anatomic site, age, or environmental conditions (Marcy et al., 2004). In our study, the incidence of fever was approximately the same after the three doses of DTwP, while only 10,8 \% of the cases with fever occurred after a booster dose administration of DTwP vaccine. Our results about fever rates after DTwP administration are consistent with the results from Braun et al. who found that fever was the most commonly reported event for DTwP, occurring in 851 of 1939 infants (43.9 \%) (Braun et al., 2000). While the incidence of fever increases substantially after the first dose $(37 \%$ of the cases), this is in disagreement with the results of Multicenter Acellular Pertussis Trial in which the incidence and severity of fever increased substantially with successive doses of the reference whole-cell vaccine (Decker \& Edwards, 1996).

\section{Discussion}

In this study, the cases reported with encephalopathy, anaphylaxis and temperature over $40{ }^{\circ} \mathrm{C}$ were not investigated and there were not made search for other causes, therefore conclusions about their relationship with DTwP combined vaccines cannot be reached.

Studies have shown that the reactogenicity to $\mathrm{wP}$ containing vaccines is significantly reduced when given in early short time-frame schedules (Miller et al., 1997). The Albanian immunization schedule is well studied and designed according to World Health Organization recommendations which is reflected also in the rates of adverse events following DTwP vaccines. Rates of adverse events over the years did not changed significantly except the second year after the implementation of AEFI reporting system and the second year after the introduction of the Pentavalent vaccine formulation. Although it is difficult to evaluate and specifically attribute adverse events to a single vaccine after the administration of combined vaccines, the introduction in 2009 of the combined Pentavalent vaccine in Albania did not increase the rate and the nature of adverse events observed before 2009 when DTwP was administered alone to children. This is consistent with the Cochrane review in 2012 which found that the use of combined versus separate components of 
the vaccine did not increase the incidence of serious adverse events RR.0,94. (CI: 0.58-1.53) (Bar-On et al., 2012).

Studies aimed at evaluate the relationship between the site of injection and the incidence of adverse reactions in DTwP recipients concluded that the administration of vaccine in the buttock elicited less local and systemic reactions than the injection in the thigh (Baraff et al., 1984). Administration of aluminum-absorbed vaccines into subcutaneous tissue instead of muscle has been associated with a higher rate of local reactions as aluminum hydroxide crystals may remain at the injection site possibly creating local inflammation and sterile abscess (Bergeson, Singer \& Kaplan, 2004 ; Jefferson, Rudin \& Di Pietrantonj, 2004). This analysis is limited because we do not have, for every recorded AEFI, the number of vaccine lot and the brand name of the manufacturer. Furthermore, the injection site is not known and the immunization method and technique of administration are also not available.

Because DTwP containing vaccines are administered to children at an age when disorders like encephalopathy, infantile spasms, neurological conditions, and SIDS are most likely to occur, it is possible that they occur after the administration of DTwP containing vaccines although the causes might be unrelated to the vaccine or vaccination. Studies have disseminated concerns about relationship between DTwP and such conditions (Howson \& Fineberg, 1992). Although wP vaccine are gradually replaced by aP vaccine in the industrialized world, the health and economic impact should be evaluated before the introduction of a new vaccine and the shift in formulation should be driven by safety considerations. Recent mathematical modeling studies from Australia, England and Wales and USA indicate that interchanging from wP to aP may be associated with disease resurgence after a few years which might also increase the risk of deaths from B. pertussis infection (Tozzi et al., 1998). In the pertussis position paper, WHO advises that National Immunization Programs currently administering wP vaccines should continue to use wP vaccines for primary vaccination series (WHO, 2016). Indeed, experiments performed in the baboon model, the aP vaccines protected against the disease but had limited impact on transmission of the agent to other animals, whereas DTwP vaccines are effective in preventing infection and transmission (Warfel \& Merkel, 2014). Besides all, when used in combination with inactivated whole-cell B. pertussis vaccine, the humoral immune response to diphtheria and tetanus toxoid is ehanced because of the intrinsic adjuvant properties of whole-cell B. pertussis.

Recent data indicate that B. pertussis strains have evolved and changed the genomic sequence of virulence factors over time, with isolates differing in pre and post vaccination era (Korkmaz1, Aydin \& Unal, 2014). An improved form of DTwP with reduced endotoxin content has been 
produced for more than 30 years by the Instituto Butantan in Brazil, and 30 million doses have been administrated to Brazilian children every year, with over 95 \% coverage without severe adverse events reported (Dias et.al., 2012). It may be interesting to evaluate the possible benefits of introducing a similar type of vaccine also in our country. During the last years, the reporting rate has reached a constant level which confirms that Albanian surveillance system of adverse events after children vaccination is wellfunctioning. The health workers are aware of the importance of reporting any adverse event, and also the measures and legal provisions undertaken by the Ministry of Health through the Institute of Public Health are well functioning leading to the establishment of a AEFI Surveillance System in accordance with the World Health Organization directives.

\section{Conclusion}

The overall rates of adverse events after administration of wP containing vaccines in Albanian children were within the range expected in WHO DTwP vaccine rates information sheet. Also the nature and pattern of reactions were not severe and the numbers of serious reactions were significantly low. Other severe events reported in DTwP rates information sheet like Hypotonic Hyporesponsive episode, Davret syndrome, aseptic meningitis, brachial neuritis, sudden infant death syndrome, Reyes syndrome, epilepsy, chronic neurological damage, hemolytic anemia, juvenile diabetes, peripheral mononeuropathy or thrombocytopenia were not reported. In this analysis most of the events occur after the first administered dose, and they start to fall after the second dose reaching a minimum level after the booster dose which is inconsistent with the reports from WHO (WHO, 2014). This fact might reflect the underreporting trend and the trend not to report mild and common reactions which increase with increasing doses of the vaccine. Anyway underreporting is not at concerning levels since the rates of AEFI fall within the expected limits stabilized by WHO. Moreover, among children, lower diphtheria toxoid content generally results in a decreased reactogenicity of booster doses (WHO, 2000).

In our study several brands of Pentavalent vaccine were reported. but limited data suggest no interchangeability between different products of vaccines as the differences in adverse reaction rates may be due to differences in DTwP vaccine preparations, antigenic content, methods and lots of production (Baraff et al., 1984).

All these observations lead to the conclusion that the DTwPcontaining vaccines employed in Albania do not raise relevant concerns about safety. Nevertheless it should be emphasized that the small number of cohorts and the rarity of this events makes it difficult the occurrence and detection of this events in a small country like Albania. 


\section{References:}

1. Australian Government . (2008). Department of Health Additional reports - childhood immunisation coverage. Commun Dis Intell 32, 288-9. Retrieved from http://www.health.gov.au/internet/main/publishing.nsf/Content/cdacdi3304a.htm

2. Baraff , L.J., Cody, C.L., \& Cherry, J.D. (1984). DTP-associated reactions: an analysis by injection site, manufacturer, prior reactions, and dose. Pediatrics, 73, 31-36. Available from http://pediatrics.aappublications.org/content/73/1/31.long

3. Bar-On ES, Goldberg, E., Hellmann, S., \& Leibovici, L .(2012). Combined DTP-HBV-HIB vaccine versus separately administered DTP-HBV and HIB vaccines for primary prevention of diphtheria, tetanus, pertussis, hepatitis B and Haemophilus influenzae B (HIB). Retrieved from Cochrane Database of Systematic Reviews, (4):CD005530. doi: 10.1002/14651858.CD005530.pub3.

4. Beigel, J., Kohl, K.S., Khuri-Bulos, N., Bravo, L., Nell, P., Marcy, S.M., ... Maurer, T. (2007). Rash including mucosal involvement: Case definition and guidelines for collection, analysis, and presentation of immunization safety data. Vaccine , 25, 5697-5706, doi:10.1016/j.vaccine.2007.02.066

5. Bergeson, PS., Singer, SA., \& Kaplan, AM.(1982). Intramuscular injections in children. Pediatrics, 70(6), 944-8. Retrieved from http://pediatrics.aappublications.org/content/70/6/944.long?sso=1\&ss o_redirect_count $=1 \&$ nfstatus $=401 \&$ nftoken $=00000000-0000-0000$ $0000-$ 000000000000\&nfstatusdescription=ERROR\%3a+No+local+token

6. Bohlke K; Davis R.L., Marcy S.M., Braun M.M., DeStefano F., Black S.B., Mullooly J.P., \& Thompson R.S. (2003). Risk of anaphylaxis after vaccination of children and adolescents. Pediatrics, 112(4), $815-20$.

7. Bonhoeffer, J., Imoukhuede, E.B., Aldrovandi, G., Bachtiar, NS., Chan, ES., Chang, ... Heininger U.(2013). Template protocol for clinical trials investigating vaccines--focus on safety elements. Vaccine, 31(47), 5602-20. doi: 10.1016/j.vaccine.2013.02.041.

8. Bouchama, A., \& Knochel, JP. (2002). Heat stroke. $N$ Engl J Med, 346, 1978- 88.

9. Braun, MM., Mootrey, GT., Salive, ME., Chen, RT., \& Ellenberg, SS. (2000). "Infant immunization with acellular pertussis vaccines in the United States: assessment of the first two years' data from the Vaccine Adverse Event Reporting System (VAERS)”. Pediatrics, 
106(4)

E51.

Retrieved

from

http://pediatrics.aappublications.org/content/106/4/e51.long

10. Cody, C.L., Baraff, L.J., Cherry, J.D., Marcy S.M., \& Manclark C.R. (1981). "Nature and rates of adverse reactions associated with DTP and DT immunizations in infants and children”. Pediatrics, 68, 650660 .Retrieved from http://pediatrics.aappublications.org/content/68/5/650.long

11. Decker, M.M., Edwards, K.M., \& Bogaerts, H.H. (2008). Combination Vaccines. In Plotkin, SA., Orenstein, WA. eds. Vaccines, 5th ed. Philadelphia, PA, WB Saunders Company, 139156. ISBN13: 978-1416036111

12. Decker, MM., \& Edwards, KM (1996). The multicenter acellular pertussis trial: An overview. Journal of Infectious Diseases, 174, supl 3, S270-S275. Retrieved from http://jid.oxfordjournals.org/content/174/Supplement_3/S270.long

13. Deloria, M.A., Blackwelder, W.C., Decker M.D., Englund J.A., Steinhoff M.C., Pichichero M.E. ... Edwards K.M. (1995). Association of reactions after consecutive acellular or whole-cell pertussis vaccine immunizations. Pediatrics, 96(3 pt 2), 592-594. Retrieved from http://pediatrics.aappublications.org/content/96/3/592.long?sso=1\&ss o_redirect_count $=1 \&$ nfstatus $=401 \&$ nftoken $=00000000-0000-0000$ 0000 000000000000\&nfstatusdescription=ERROR\%3a+No+local+token

14. Dias, W.O., van der Ark, A.A.J, Sakauchi, M.A., Kubrusly, F.S., Prestes, A.F.R.O., Monamaris ... Isaias R. (2013). An improved whole cell pertussis vaccine with reduced content of endotoxin. Human Vaccines \& Immune-therapeutics 9(2), 339-348. doi: $10.4161 / \mathrm{hv} .22847$

15. Fenichel, GM. (1982). "Neurological complications of immunization". Annals of Neurology, 12(2), 119-28. doi: 10.1002/ana.410120202

16. Gidudu , J.(2008). A local reaction at or near injection site: Case definition and guidelines for collection, analysis, and presentation of immunization safety data. Vaccine, 26, 6800-6813, doi:10.1016/j.vaccine.2008.10.006

17. Gold, M., Kempe, A., \& Osbourn, M. (1999). “A comparison of serious adverse reactions to whole cell and acellular pertussis vaccines in South Australia”. Med J Aust, 171(6), 331-Available from

https://www.ncbi.nlm.nih.gov/pubmed/?term=\%E2\%80\%9CA+comp arison+of+serious+adverse+reactions+to+whole+cell+and+acellular+ 
pertussis+vaccines+in+South+Australia\%E2\%80\%9D.++Med+J+Au st

18. Greco, D., Salmaso, S., Mastrantonio, P., Giuliano, M., Tozzi , AE., ... Anemona, A. (1996). A controlled trial of two acellular vaccines and one whole-cell vaccine against pertussis. Progetto Pertosse Working Group. N Engl J Med , 334(6), 341-8. doi: 10.1056/NEJM199602083340601, Retrieved from http://www.nejm.org/doi/full/10.1056/NEJM199602083340601

19. Howson, C.P., Fineberg, H.V. (1992). Adverse events following pertussis and rubella vaccines: summary of a report of the Institute of Medicine. JAMA, 267(3), 392-396. doi:10.1001/jama.1992.03480030070039.

20. Institute of Medicine (U.S.). Committee to Review the Adverse Consequences of Pertussis and Rubella Vaccines (1991). Adverse Effects of Pertussis and Rubella Vaccines: A Report of the Committee to Review the Adverse Consequences of Pertussis and Rubella Vaccines. Washington, D.C. : National Academy Press, 1991 . ISBN: 0309044995

21. Institute of Medicine. (1991). "Adverse effects of vaccines: Evidence bearing on causality”. The National Academies Press, Washington, DC. Available from https://www.nap.edu

22. Jefferson, T., Rudin, M., \& Di Pietrantonj, C.(2004). Adverse events after immunisation with aluminium-containing DTP vaccines: systematic review of the evidence. Lancet Infect Dis, 4, 84-90. http://dx.doi.org/10.1016/S1473-3099(04)00927-2

23. Johansson, SG., Hourihane, JO., Bousquet, J., Bruijnzeel-Koomen, C., Dreborg, S., ... Haahtela, T.(2001). A revised nomenclature for allergy. An EAACI position statement from the EAACI nomenclature task force. Allergy, 56(9), 813-24. doi: 10.1111/j.13989995.2001.00002.x-i1

24. Kohl, K.S., Ball, L., Gidudu, J., Hammer, S.J., Halperin, S., Heath, P., ... Walop, W. (2007). Abscess at injection site: Case definition and guidelines for collection, analysis, and presentation of immunization safety data. The Brighton Collaboration Local Reactions Working Group for Abscess at Injection Site. Vaccine , 25, 5821-5838. doi: 10.1016/j.vaccine.2007.04.057

25. Korkmaz1, H.A., Aydin, A., \& Unal, B.(2014). Comparison of acellular pertussis-tetanusdiphtheria vaccines and whole-cell pertussistetanus- diphtheria vaccines in infancy. Paediatr Int Child Health, 34(3), 198-202. doi: 10.1179/2046905513Y.0000000110.

26. Marcy, S.M.,Kohl K.S., Dagan, R., Nalin, D., Blum, M., Jones, M.C., ... Vermeer, P. (2004). Fever as an adverse event following 
immunization: case definition and guidelines of data collection, analysis, and presentation. Vaccine, 22: 551-556, doi:10.1016/j.vaccine.2003.09.007

27. Miller E., Ashworth L.A., Redhead K., Thornton C., Waight P.A., \&Coleman T. (1997). "Effect of schedule on reactogenicity and antibody persistence of acellular and whole-cell pertussis vaccines: value of laboratory tests as predictors of clinical performance". Vaccine, 15, 51-60.Retrieved from http://www.sciencedirect.com/science/article/pii/S0264410X9600112 0

28. Mortimer, E. Pertussis. In: Plotkin, S., Mortimer, E., et al.,. Vaccines. 2nd ed. Philadelphia, PA: WB Saunders; 1994.

29. Pollock, TM., Miller, E., Mortimer, JY., \& Smith, G. (1984). "Symptoms after primary immunization with DTP and DT vaccine". Lancet, 2, 146-149. http://dx.doi.org/10.1016/S0140-6736(84)910572

30. Rantala, H., \& Uhari, M.(1989). Occurrence of childhood encephalitis: A population- based study. Pediatric Infect Dis J8, 426-430.Available from https://science.report/pub/16604795

31. Ray, P., Hayward, J., Michelson, D., David M.D., Lewis, E., Schwualbe, J., ... Robert M.D. (2006). Encephalopathy after WholeCell Pertussis or Measles Vaccination Lack of Evidence for a Causal Association in a Retrospective Case-Control Study. The Pediatric Infectious Disease Journal, 25(9), 768-73. doi: 10.1097/01.inf.0000234067.84848.e1

32. Rüggeberg J.U, Gold M.S., Bayas J.M., Blum M.D., Bonhoeffer. J., Friedlander.S., ... Simpson N.(2007). "Anaphylaxis: Case definition and guidelines for data collection, analysis, and presentation of immunization safety dat”. Vaccine, 25(31), 5675-84. doi:10.1016/j.vaccine.2007.02.064

33. Sejvar, J. J., Kohl, K. S., Bilynsky, R., Blumberg, D., Cvetkovich, T., Galama, J., ... null, null (2007). Encephalitis, myelitis, and acute disseminated encephalomyelitis (ADEM): Case definitions and guidelines for collection, analysis, and presentation of immunization safety data. Vaccine, 25(31), 5771-5792. DOI: 10.1016/j.vaccine.2007.04.060

34. Tozzi A.E., CiofidegliAtti, M.L., FiteWassilakj, S.G., Salmaso, S., Panei, P., Anemona, A., Luzi, S., \& Greco, D. (1998). Predictors of adverse events after the administration of acellular and whole cell diphtheria-tetanus-pertussis vaccines. Vaccine, Vol. 16, 320322,.Available from https://www.ncbi.nlm.nih.gov/pubmed/?term=Predictors+of+adverse 
+events+after+the+administration+of+acellular+and+whole+cell+dip htheria-tetanus-pertussis+vaccines.

35. Warfel, J.M., \& Merkel, T.J. (2014). Reply to Domenech de Celles et al.: Infection and transmission of pertussis in the baboon model. Proc Natl Accad Ssci USA, 111(7), E718 doi: 10.1073/pnas.1324074111

36. World Health Organisation. (2016, March 14). Pertussis vaccines: WHO position paper, August 2015-Recommendations Vaccine, 34(12),1423-1425. http://dx.doi.org/10.1016/j.vaccine.2015.10.136

37. World Health Organization .(2014). Information Sheet, Observed rate of vaccine reactions - Diphtheria, pertussis, tetanus vaccines. Retrieved from http://www.who.int/vaccine_safety/initiative/tools/vaccinfosheets/en/ . Last seen on August 2016.

38. World Health Organization. Dept. of Vaccines and Biologicals. (2000). "Supplementary information on vaccine safety, Part 2: Background rates of adverse events following immunization". Geneva : World Health Organization. Retrieved from http://www.who.int/iris/handle/10665/66675 